\title{
Optimized Effective Potentials in Finite Basis Sets
}

\author{
Tim Heaton-Burgess, ${ }^{1}$ Felipe A. Bulat, ${ }^{1}$ and Weitao Yang $^{1}$ \\ ${ }^{1}$ Department of Chemistry, Duke University, \\ Durham, North Carolina 27708, USA
}

(Dated: September 16, 2018)

\begin{abstract}
The finite basis optimized effective potential (OEP) method within density functional theory is examined as an ill-posed problem. It is shown that the generation of nonphysical potentials is a controllable manifestation of the use of unbalanced, and thus unsuitable, basis sets. A modified functional incorporating a regularizing smoothness measure of the OEP is introduced. This provides a condition on balanced basis sets for the potential, as well as a method to determine the most appropriate OEP potential and energy from calculations performed with any finite basis set.
\end{abstract}

PACS numbers: 31.10.+z, 31.15Ew, 31.15.Pf 
Kohn-Sham density functional theory (KS DFT) [1] enjoys wide application owing to the computational accessibility afforded by formulating the many body problem in terms of the noninteracting KS reference system. However, the exact exchange-correlation energy functional $E_{x c}$ and corresponding local potential, $v_{x c}$, are unknown. The future success of DFT is dependent on the availability of suitable approximations for $E_{x c}$. Significant interest is being shown for the development of implicit density functionals, depending explicitly on the KS orbitals [2]. For such functionals, $v_{x c}$ cannot be directly obtained as a simple functional derivative and requires an OEP method for its determination [3, 4, 5].

The OEP concept first appeared within the Hartree-Fock (HF) formalism [6], and later was employed in DFT [7] using the HF exact exchange energy functional (EXX). These conventional approaches identify the local KS potential for exact exchange as that obtained from solving $\delta E\left[\left\{\phi_{i}^{\mathrm{KS}}\right\}\right] / \delta v_{s}(\mathbf{r})=0$, which in turn leads to a linear integral equation to be solved for the OEP. The rigorous justification of the OEP method as a variational minimization has however only recently been provided within the potential functional formulation [8]. This formulation lends itself to a direct approach to solving for the OEP [5] where the minimization of the energy functional over local potentials is considered.

The discrete representation of the OEP problem in finite basis sets for both the KohnSham orbitals and the potential can be ill-posed [9, 10]: While the total OEP energy is stable with respect to the changes in the potential, there can be many different potentials, including nonphysical ones, having numerically degenerate total energies. This has lead to some degree of confusion of what in fact constitutes a valid, finite basis, OEP implementation [10], when the ill-posedness is not appropriately accounted for.

In this Letter, we will show that the ill-posed nature of the discrete OEP originates from the use of basis sets that are unsuitable, as a result of being unbalanced. This ill-posedness does not imply that the OEP method is unphysical by construction, rather, just that it can lead to nonphysical potentials. The regularization method developed in this work insures that the physical context of finite basis OEP calculations are maintained in all cases, with generation of physically meaningful potentials.

The following analysis reveals the physical origin of the ill-posed nature in the discrete OEP problem. In determining the OEP, one looks for the total-energy minimizing potential $v_{s}(\mathbf{r})$ whose lowest $N$ eigenstates are the set of wavefunctions with the finite basis set expansion, $\left|\phi_{i}\right\rangle=\sum_{\mu} c_{i \mu}\left|\chi_{\mu}\right\rangle$, for $i=1, \ldots, N$, satisfying the one-electron equations 
$\sum_{\mu}\left\langle\chi_{\nu}\left|-\frac{1}{2} \nabla^{2}+v_{s}(\mathbf{r})\right| \chi_{\mu}\right\rangle c_{i \mu}=\epsilon_{i} \sum_{\mu}\left\langle\chi_{\nu} \mid \chi_{\mu}\right\rangle c_{i \mu}$. This equation is the only link from $v_{s}(\mathbf{r})$ to the OEP energy. If a variation of $v_{s}(\mathbf{r})$ is outside of the range of the orbital basis set $\left\{\chi_{\mu}\right\}$ - meaning such a variation in the potential leads to numerically the same total energy and orbitals $\left|\phi_{i}\right\rangle$ - then this variation can lead to a nonphysical potential. The nonphysical potentials reported in Ref. [10] appear to come from such a construction. For nonphysical potentials with wild oscillations, the orbital basis set is certainly inadequate for solving the one-electron equations and the discrete OEP equation is a poor approximation to the original OEP problem. Given an orbital basis set $\left\{\chi_{\mu}\right\}$, there are many variations of $v_{s}(\mathbf{r})$ that can lead to such behavior, one example being adding to $v_{s}(\mathbf{r})$ Gaussian functions with exponents much higher than the exponents in the orbital basis set $\left\{\chi_{\mu}\right\}$. We consider such a potential basis set unbalanced with the orbital basis set.

While a careful choice of basis sets for the orbitals and the potential can lead to accurate potentials, as shown in many previous calculations [4, 5, 9], we aim here to develop a robust method which can deliver accurate OEP potentials and energies, with both balanced and unbalanced basis sets.

To examine the reliability of our approach we use the OEP procedure with the LDA (SVWN5) functional so that a direct comparison between the calculated OEP potentials and the LDA potential can be made. The success of our method for LDA OEP transfers to EXX as will be shown.

Our OEP implementation is the direct optimization approach of Yang and $\mathrm{Wu}$ [5] where the trial potential is expanded in a finite basis set, $\left\{g_{t}\right\}$, as $v_{s}^{\sigma}(\mathbf{r})=v_{\text {ext }}(\mathbf{r})+v_{0}(\mathbf{r})+\sum_{t} b_{t}^{\sigma} g_{t}(\mathbf{r})$. Here $v_{\text {ext }}$ is the external potential of the system under consideration and $v_{0}$ is a fixed reference potential, taken as the Fermi-Amaldi potential (or the Coulomb potential for LDA) for the sum of the atomic densities so to enforce the correct asymptotic behavior upon $v_{s}$. Transferring the functional dependence from the KS potential on to the expansion coefficients $\left\{b_{t}^{\sigma}\right\}$ in this way gives rise to an efficient implementation of the OEP based on the unconstrained minimization of $E\left(\left\{b_{t}^{\sigma}\right\}\right)$ with readily available analytic derivatives [5]. The iterative minimization of $E\left(\left\{b_{t}^{\sigma}\right\}\right)$ is efficiently achieved using an approximate Newton method [11] which takes an approximate form for the Hessian as $H_{u t}^{\sigma}=\sum_{i a} \frac{\left\langle\phi_{a \sigma}\left|g_{u}\right| \phi_{i \sigma}\right\rangle\left\langle\phi_{a \sigma}\left|g_{t}\right| \phi_{i \sigma}\right\rangle}{\epsilon_{i \sigma}-\epsilon_{a \sigma}}$.

In the following we will consider two forms of regularization for extracting physical potentials from ill-posed finite basis OEP calculations. The first, used by us before [5], 11], however will be shown to be inadequate to remove all irregularities in the resulting potential. 
We then introduce a new form of regularization for the OEP that produces potentials of the highest quality, along with the corresponding energy.

We will focus on the specific example of argon with the orbital basis cc-pVDZ and consider the effects of different potential basis on the potentials and energies obtained from the OEP procedure. The specific basis sets we use for the potential are the orbital basis itself, and three s-type even tempered (ET) basis sets denoted as ArX (X=64,1024,8192) with exponents $2^{n},-4 \leq n \leq \log _{2}(\mathrm{X})$.

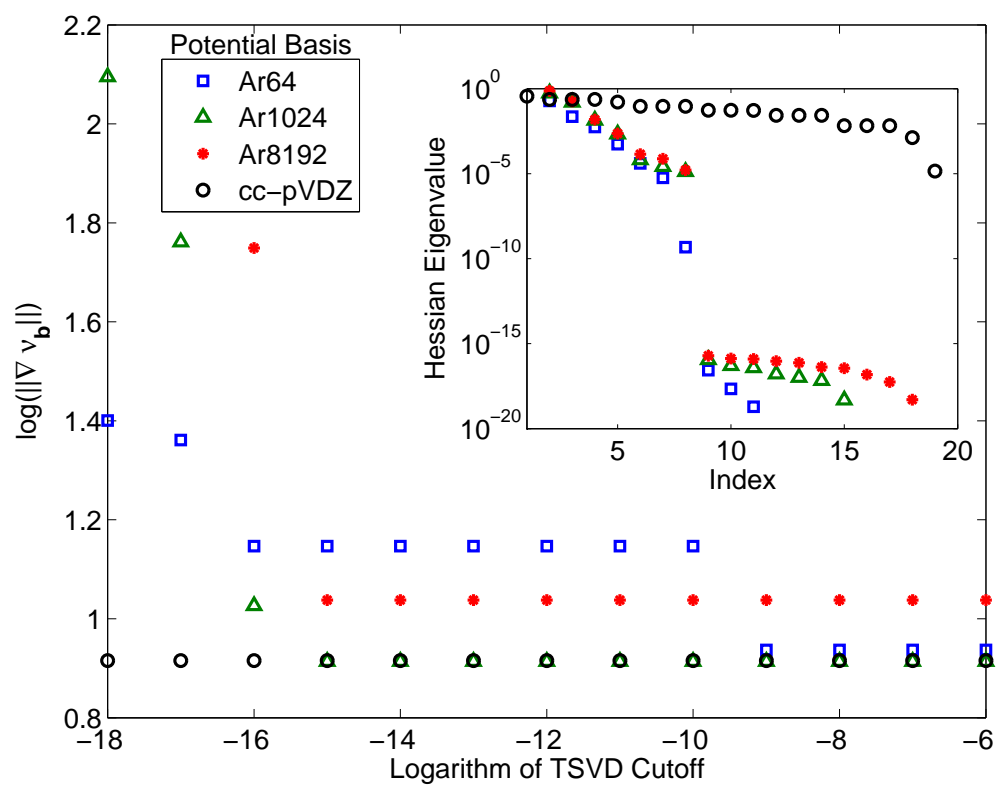

FIG. 1: Variation in the smoothness of the LDA OEP potential as a function of the SVD cutoff for argon (orbital basis cc-pVDZ) with four different potential basis sets. Insert: Spectrum of the approximate Hessian for each basis set.

The nature of the Hessians spectrum is intimately related to the behavior of the potentials obtained. Most applications of the direct OEP procedure have made use of coinciding orbital-quality basis sets for both orbitals and potential. This, generally, generates physical potentials and energies. From the Hessian spectrum in Fig. 1 we see that using the cc-pVDZ orbital and potential basis leads to a full rank Hessian resulting in the convergence of the OEP to a physical potential. However, if we use other constructions for the potential basis such as ET basis sets, we can observe much different spectral structures for the Hessian. The rank deficiency for the three ET basis sets is obvious from the significant gap in the spectra of Fig. 1. To solve the update equation in an stable manner, both truncated 


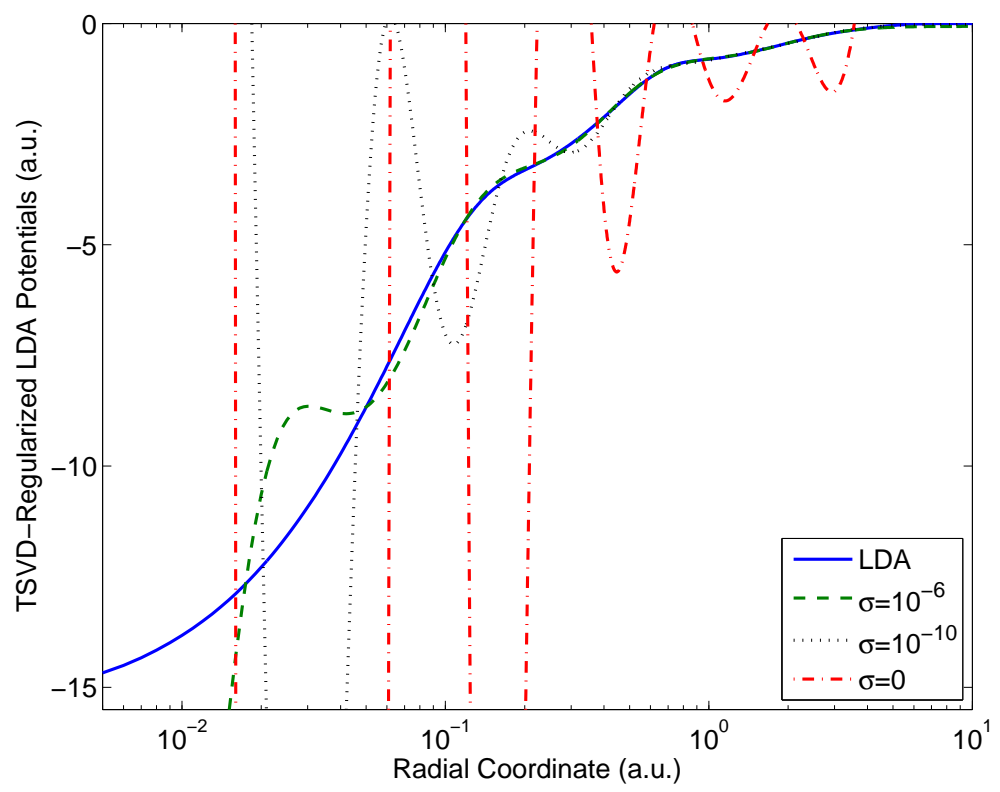

FIG. 2: TSVD-regularized LDA potentials of argon for various values of the SVD cutoff, $\sigma$, using the potential basis Ar8192. The solid line depicts the exact LDA potential for comparison.

SVD (TSVD) and Tikhonov regularizations have been proposed and implemented [11]. The TSVD regularization, to be used in this discussion, neglects contributions from eigenvectors corresponding to those singular values below some chosen cutoff. Fig. 1 shows how the smoothness of the resulting OEP, as measured by the norm $\left\|\nabla \sum_{t} b_{t} g_{t}(\mathbf{r})\right\|$, varies as a function of the TSVD cutoff used when solving the Hessian update equation. As the cutoff approaches the lower cluster of singular values, the structure becomes step-like, with jumps in the norm as the cutoff passes through eigenvalues of the Hessian. The potential becomes very nonphysical (Fig. 2), reminiscent of those displayed in Ref. [10]. It is clear that this TSVD regularization, on its own, is not sufficient to ensure a physically meaningful solution for the OEP in general. It does however confine irregularities to regions near the nuclei, which could be sufficient in many applications.

Next, we take the classical approach to ill-posed problems and further incorporate some desirable measure to regularize the solution [12]. It is clear that any nonphysical oscillatory behavior in the potential will be confined to the basis set expansion $v_{\mathbf{b}}=\sum_{t} b_{t} g_{t}$. We thus introduce a $\lambda$-regularization by constraining our solutions to yield smooth potentials as measured by the smoothing norm $\left\|\nabla v_{\mathbf{b}}\right\|$, thereby restricting the nonphysical variations in the potential because of unbalanced basis set. This norm is certainly not unique, however 
is simple to implement and will be seen to produce very satisfying results. We define a regularized energy functional as

$$
\Omega_{\lambda}(\mathbf{b})=E^{\mathrm{YW}}(\mathbf{b})+\lambda\left\|\nabla v_{\mathbf{b}}(\mathbf{r})\right\|^{2},
$$

where $\left\|\nabla v_{\mathbf{b}}(\mathbf{r})\right\|^{2}=2 \mathbf{b}^{\mathrm{T}} \mathbf{T} \mathbf{b}, \mathbf{T}$ is the kinetic energy integral matrix in the potential basis and $E^{\mathrm{YW}}(\mathbf{b})$ is the OEP energy calculated according to the Yang-Wu method. The energy derivatives with respect to the coefficients are modified accordingly as $\nabla_{\mathbf{b}} \Omega_{\lambda}=\nabla_{\mathbf{b}} E^{\mathrm{YW}}+$ $4 \lambda \mathbf{T b}$ and $\nabla_{\mathbf{b}}^{2} \Omega_{\lambda}=\nabla_{\mathbf{b}}^{2} E^{\mathrm{YW}}+4 \lambda \mathbf{T}$. The significance of the smoothing contribution relative to the energy minimization is then determined by the magnitude of $\lambda$. Our method of determining the most appropriate value of $\lambda$ (that is, the value of $\lambda$ which corresponds to a physical potential) is the classical L-curve analysis [12]. This involves constructing the plot of $\log \left(\left\|\nabla v_{\mathbf{b}}\right\|\right)$ against $\log \left(E^{\mathrm{YW}}-E^{\mathrm{ref}}\right)$ for $\lambda$ over some appropriate interval. The reference energy, $E^{\text {ref }}$, is taken as $E^{\mathrm{HF}}$ for EXX calculations and $E^{\mathrm{LDA}}$ for LDA OEP.

This curve presents the trade-off between the desire to extract a smooth potential, with that of an unconstrained minimization of the energy functional which can allow the introduction of nonphysical potentials. For a balanced potential basis set - defined here as one that converges to a smooth physical potential - gradually increasing $\lambda$ from zero has no significant effect on the norm $\left\|\nabla v_{\mathbf{b}}\right\|$. In such a situation no $\lambda$-regularization is needed. On the other hand, a general basis can see a significant decrease in the norm as $\lambda$ increases, until there comes a region where variations in the the norm subside corresponding to the formation of a stable and physical potential.

The most appropriate choice of $\lambda$ for any particular physical application is not always a trivial decision, dependent on the particular system under consideration and the tolerance that are acceptable [12]. In the present context, our calculations have lead to a consistent definition: The $\lambda$ corresponding to the optimal potential, and thus also the OEP energy for the given finite basis sets and chosen smoothing norm, is that point on the L-curve with minimum slope and, if it is not unique, that has the minimum value of $\left\|\nabla v_{\mathbf{b}}\right\|$. Support for such a choice is presented in [13].

Figs. 3 and 5 present such L-curve analysis for our argon example with LDA and EXX functionals. We use the notation $\mathrm{AO} / \mathrm{PB}(\lambda)$ to describe calculations performed with $\mathrm{AO}$ and $\mathrm{PB}$ atomic orbital and potential basis sets respectively, and with a regularization parameter of $\lambda$. For consistency we chose to perform the calculations without any TSVD regularization. 


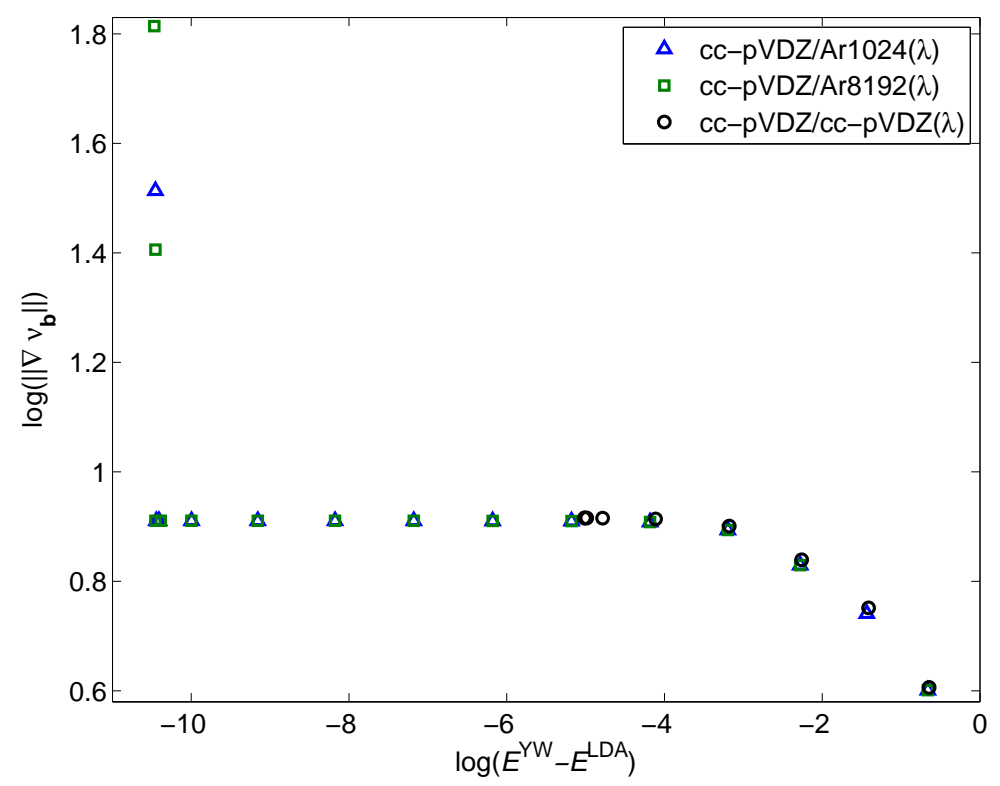

FIG. 3: Argon (cc-pVDZ) LDA OEP L-curve for various potential basis sets.

The curve for cc-pVDZ/cc-pVDZ $(\lambda)$ shows that no regularization needs to be applied to solve for an appropriate OEP as explained previously, however the cost of using such a basis is an energy higher than that can be obtained from more flexible ET basis sets. The most appropriate LDA OEP potential for this basis set (cc-pVDZ/cc-pVDZ (0)) as seen in Fig. 4 underestimates the LDA potential near the nucleus.

All three of the ET basis sets produce poor potentials with oscillations extending far from the nuclei when no regularization is used. The LDA L-curves for cc-pVDZ/Ar8192( $\lambda)$ and cc-pVDZ/Ar1024 $(\lambda)$ are of an ideal form - as $\lambda$ tends towards zero smooth potentials are generated with numerical energy agreement with the LDA value, at which point the curve rises vertically corresponding to the introduction of nonphysical oscillations in the potential. In this situation the choice of a meaningful potential is clear, the corner of the L-curve is well defined and $\lambda^{*}=10^{-14}$, the optimal choice of $\lambda$, is that point infinitesimally close to the corner (with minimum slope). The corresponding potential (Fig. 4) agrees with the LDA potential in all but a small region around the nuclei.

The EXX results for the same basis sets in Figs. 5 and 6 show that the critical value of $\lambda$ occurs at $\sim \Delta E=10^{-4}$ a.u. Significantly, this example illustrates that nonphysical potentials can be obtained when the ill-posedness is not addressed, and that the physical OEP potentials can be obtained with our method. As another example, the EXX OEP 


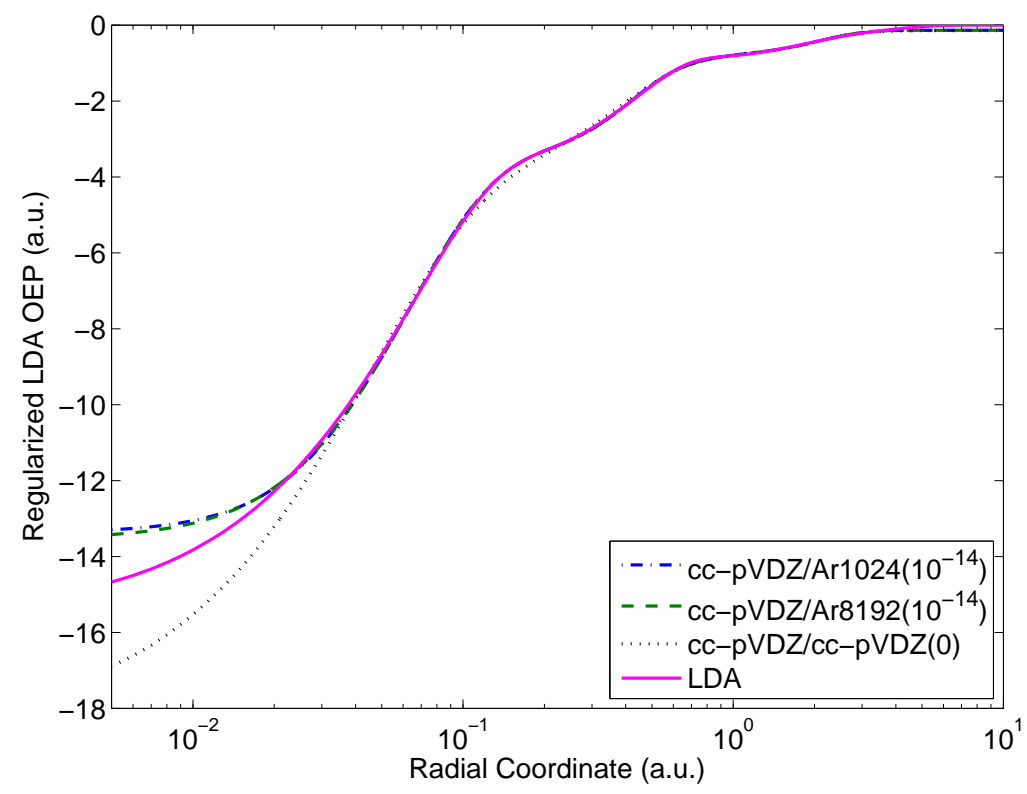

FIG. 4: Optimal LDA OEP potentials for argon (cc-pVDZ) obtained from the L-curve analysis for each basis set.

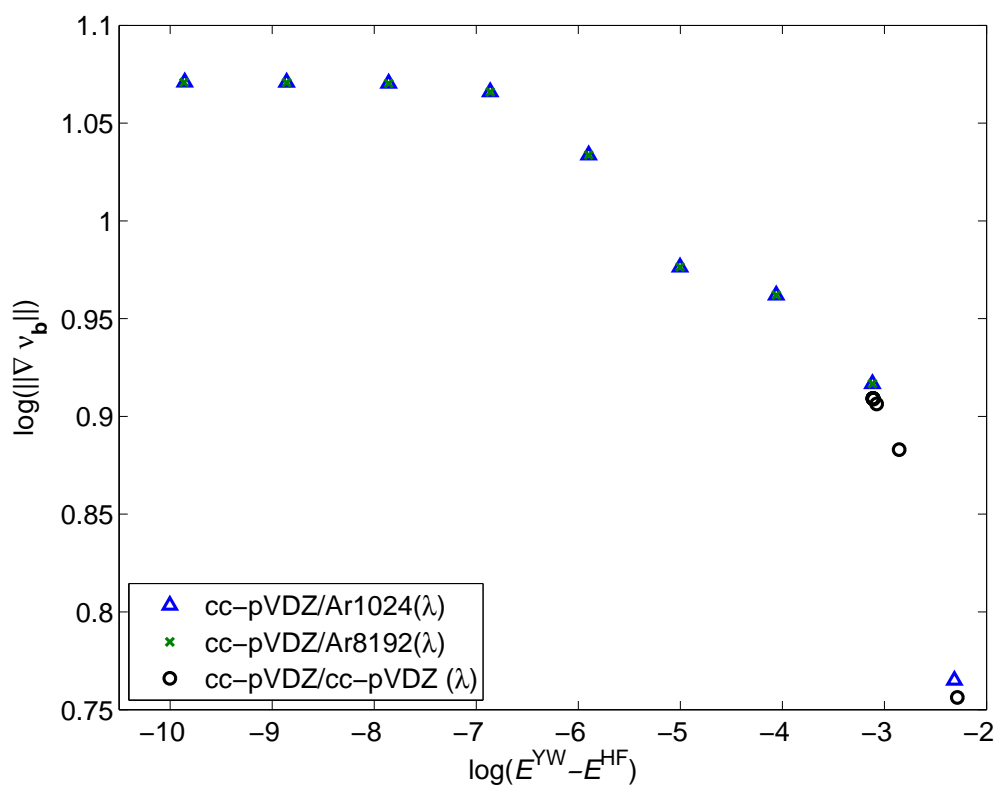

FIG. 5: Argon (cc-pVDZ) EXX L-curve for various potential basis sets.

L-curve analysis for $\mathrm{N}_{2}$ is shown in Fig. 7. We see that all basis sets regularize remarkably consistently, giving rise to a consistent OEP energy that will be explored in some detail within a future paper. Further detailed examples for atoms and molecules are provided online via EPAPS [13]. 


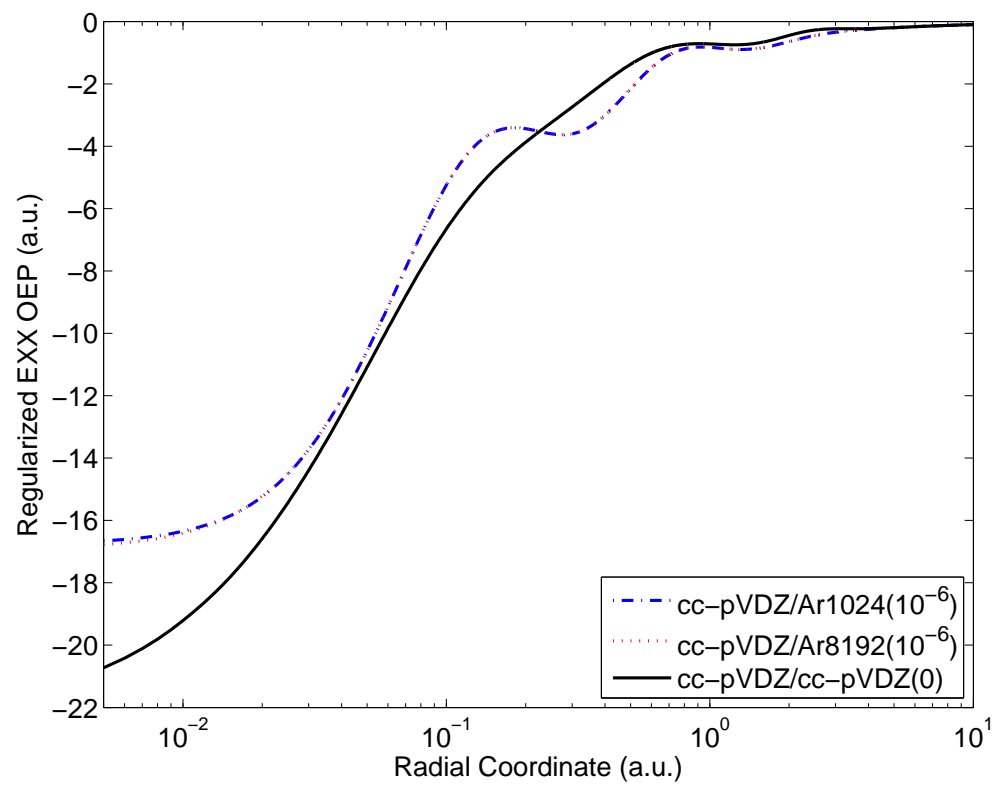

FIG. 6: Optimal EXX OEP potentials for argon (cc-pVDZ) obtained from the L-curve analysis for each basis set.

In conclusion, we have constructed a regularization procedure by introducing a measure of the smoothness of the OEP into the energy functional. This, together with an L-curve analysis, allows us to determine the physically meaningful OEP potential and energy from calculations performed with any finite basis sets. We have shown that one quantitative measure of such balance is provided by the approximate Hessian, however this does not provide an a priori judgement needed for routine application of finite basis OEP calculations. For this, the construction of balanced potential basis sets are required. Support from the National Science Foundation is gratefully acknowledged.

[1] R. G. Parr and W. Yang, Density-Functional Theory of Atoms and Molecules (Oxford University Press, Oxford, 1989).

[2] P. Mori-Sánchez, A. Cohen, and W. Yang, J. Chem. Phys. 124, 091102 (2006).

[3] S. Ivanov, S. Hirata, and R. J. Bartlett, Phys. Rev. Lett. 83, 5455 (1999).

[4] A. Görling, Phys. Rev. Lett. 83, 5459 (1999).

[5] W. Yang and Q. Wu, Phys. Rev. Lett. 89, 143002 (2002).

[6] J. D. Talman and W. F. Shadwick, Phys. Rev. A 14, 36 (1976). 


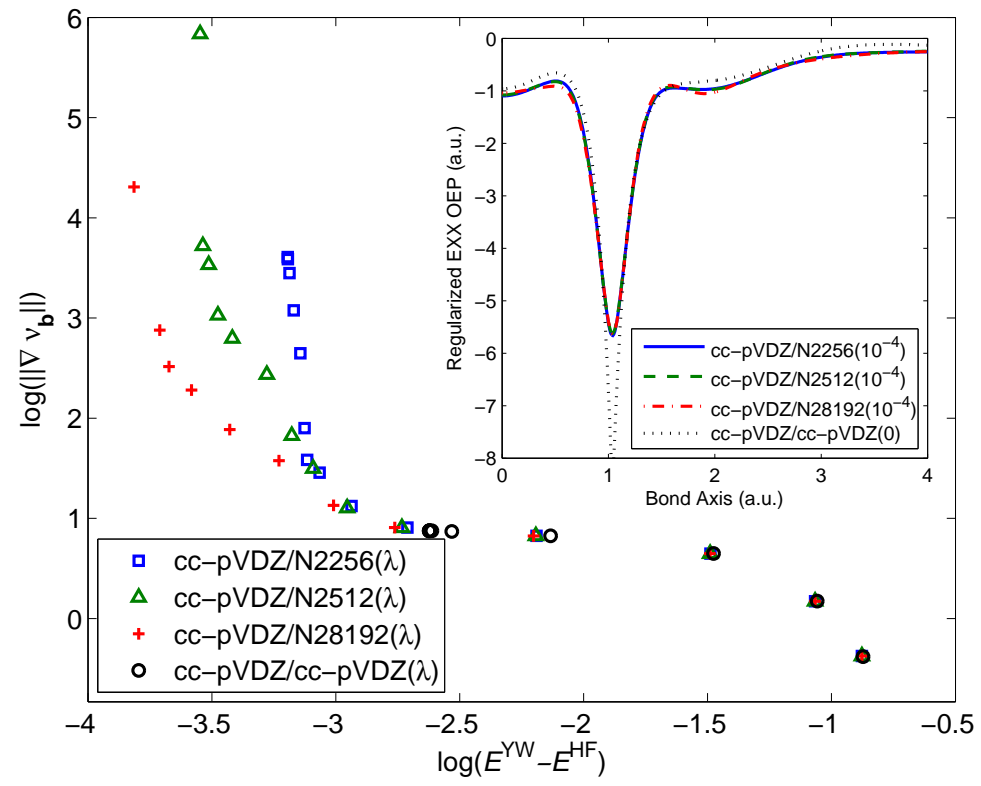

FIG. 7: EXX L-curve of $\mathrm{N}_{2}$ (cc-pVDZ) with four potential basis sets. The basis sets are defined in [13]. Insert: optimal potentials as determined by the L-curve.

[7] V. Sahni, J. Gruenebaum, and J. P. Perdew, Phys. Rev. B 26, 4371 (1982).

[8] W. Yang, P. W. Ayers, and Q. Wu, Phys. Rev. Lett. 92, 146404 (2004).

[9] S. Hirata, S. Ivanov, I. Grabowski, R. J. Bartlett, K. Burke, and J. D. Talman, J. Chem. Phys. 115, 1635 (2001).

[10] V. N. Staroverov, G. E. Scuseria, and E. R. Davidson, J. Chem. Phys. 124, 141103 (2006).

[11] Q. Wu and W. Yang, J. Theor. Comp. Chem. 2, 627 (2003).

[12] P. C. Hansen, Rank-Deficient and Discrete Ill-Posed Problems (SIAM, PA, 1989).

[13] See EPAPS Document No. ????? for further discussion and examples on $\left(\mathrm{H}_{2} \mathrm{O}\right.$ and $\mathrm{N}_{2}$ ). A direct link to this document may be found in the online articles HTML reference section. The document may also be reached via the EPAPS homepage (http://www.aip.org/pubservs/epaps.html) or from ftp.aip.org in the directory /epaps/. See the EPAPS homepage for more information. 


\section{Supplementary Material}

\section{OUR CHOICE OF THE OPTIMAL REGULARIZATION PARAMETER $\lambda^{*}$}

Firstly, we wish to consider our choice of $\lambda^{*}$ : The value of the regularization parameter corresponding to the optimal choice of potential. In many statistical applications, it is recommended to choose that corner of the L-curve with maximum curvature, for this point is argued to represent the optimal tradeoff between stability of solutions, and absolute minimization of the quantity of interest [12]. This however is dependent on the physical system under consideration and the tolerances acceptable to the user. We find the maximum curvature point on the L-curve not to be optimal for our concern - irregularities have been introduced and the regularized potential has began to diverge away from the physical potential. The over-smoothing at the corner of the L curve has also been observed in some applications [12].

The following example shows, for an LDA calculation of nitrogen (potential basis set is introduced in section III), the changes in the potential as we traverse the corner of the L-curve in Fig. (8). $\lambda=10^{-4}$ best corresponds to that point on the L-curve with minimum slope, (or minimum absolute gradient), while the maximum curvature corresponds to $\lambda=10^{-6}$. The irregularities appearing with the different $\lambda$ are most noticeable at the origin and for large $z$.

In the following we will go though several more examples showing the versatility of our procedure.

\section{WATER}

We shall consider the orbital basis of cc-pVDZ, and the three potential basis sets cc-pVDZ, H2O01, and H2O02. H2O01 and H2O02 are even tempered (ET) basis sets constructed as follows: $\mathrm{H} 2 \mathrm{O} 01$ - 9s9p $(\mathrm{O})$ and 9s $(\mathrm{H})$ uncontracted cartesian Gaussians with exponents $2^{n},-3<n<5$. H2O02 - 18s15p (O) and 18s (H) uncontracted cartesian Gaussians with exponents $2^{n},-4<n<13(\mathrm{~s})$ and $2^{n},-4<n<13(\mathrm{p})$. Our construction of the ET basis sets are such to encourage poor behavior in the resulting, unregularized, potentials so to show the versatility of our procedure. 


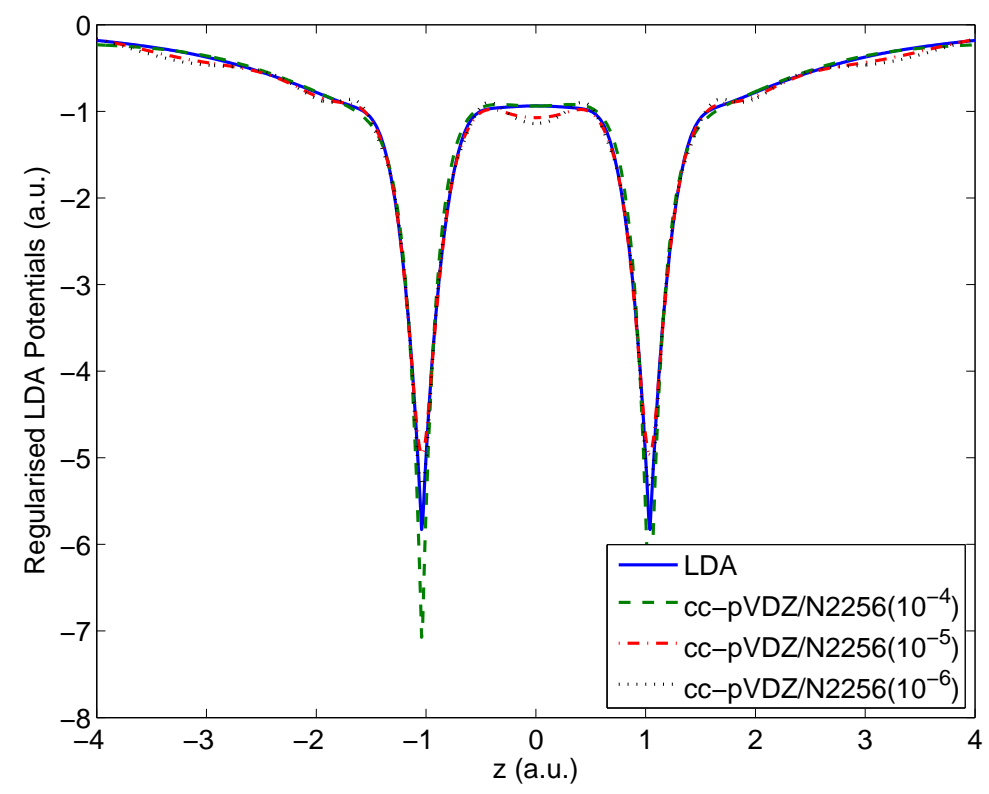

FIG. 8: LDA OEP potentials for $\mathrm{N}_{2}$ (cc-pVDZ) in the vicinity of the L-curve corner for the potential basis N2256.

We use the geometry $0.917 \AA$ for the $\mathrm{O}-\mathrm{H}$ bond length and 104.4 degrees for the $\mathrm{HOH}$ angle, and plot potentials along the $\mathrm{HOH}$ bisector.

\section{A. LDA}

For water, the Hessian spectra are shown in Fig. (91). As is typical for molecules, it becomes much harder to interpret the Hessian spectra in terms of a definable cluster of singular values that we would consider to be associated with null-space eigenvectors. The problem becomes very much ill-conditioned for the basis H2O02: the eigenvectors decay gradually to (numerically) zero, as opposed to the situation presented in the paper for Ar where the problem is very much rank deficient.

The variation of $\left\|\nabla v_{\mathbf{b}}\right\|$ as a function off the TSVD cutoff, $\sigma^{*}$, is presented in Fig. (10), in an attempt for extract a definable range of eigenvalues for which we can consider the Hessian to be stable if used in solving the update equation $\mathbf{H}\left(\mathbf{b}_{n}\right) \mathbf{p}=-\nabla E\left(\mathbf{b}_{n}\right)$. The step structure as was seen in the main text is still present, however for the H2O02 basis it becomes very hard to argue that any particular value of the TSVD cutoff would be any more favorable for a TSVD-only regularized solution for the OEP. The other two basis sets provide very clear interpretations in terms of the eigenspectrum of Fig. (9). H2O01 has a smallest eigenvalue 


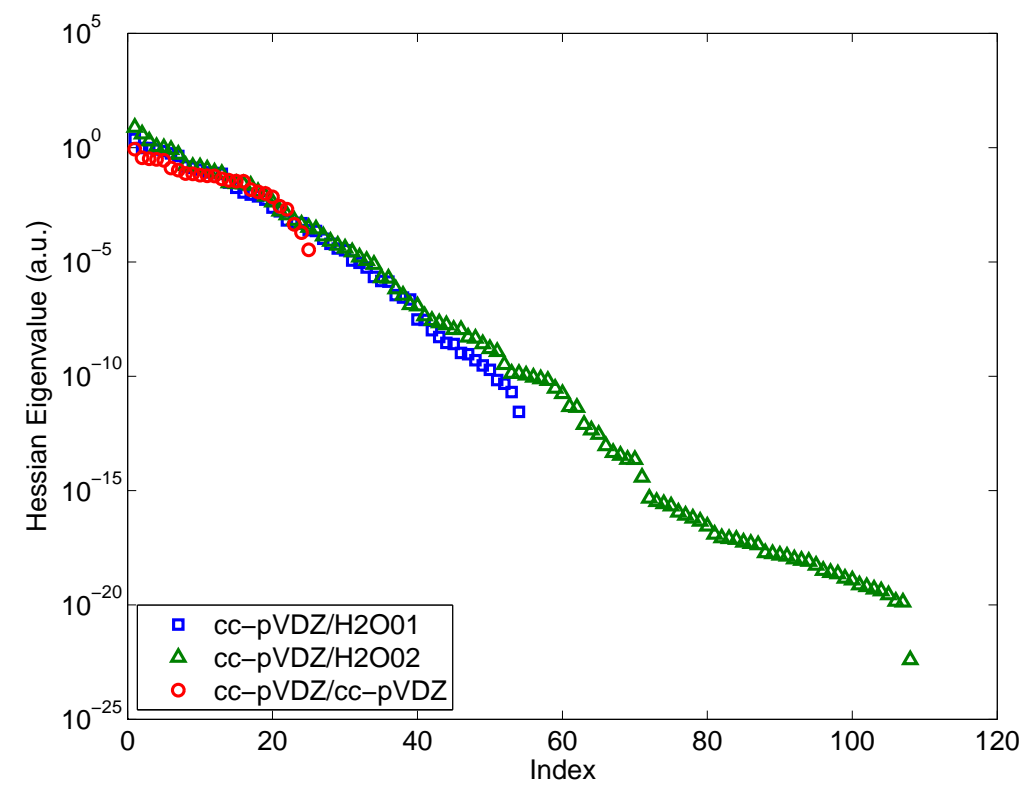

FIG. 9: Spectrum of the approximate Hessian for each basis set.

of only $3.5 \times 10^{-12}$, so that any choice for a TSVD cutoff smaller than this will yield identical results. The resulting potential is, however, very poor with nonphysical oscillations about the oxygen atom as seen in Fig. (11). Choosing a value for the cutoff around $10^{-5}$ does lead to much better potentials, but still there are some irregularities around the nucleus. The situation for cc-pVDZ/cc-pVDZ is typical, the Hessian eigenvalues decay to only $\sim 3 \times 10^{-5}$ a.u.

It is clearly necessary to apply the $\lambda$-regularization for both ET basis sets in order to produce appropriate potentials. We chose not to apply any TSVD when solving the update equation and obtain the L-curves seen in Fig. (12), in turn giving the potentials, corresponding to the minimum slope point, in Fig. (13)

\section{B. EXX}

The Hessian structure is very similar to that of the LDA case. This is a very general observation for all the systems we have examined, so the discussion for the LDA case carries over to EXX. As such we will specifically only discuss the L-curves and the potentials we can extract in the EXX case although a complete set of figures are presented.

The L-curves of Fig. (17) agree exceptionally well for all but the smallest amounts of 


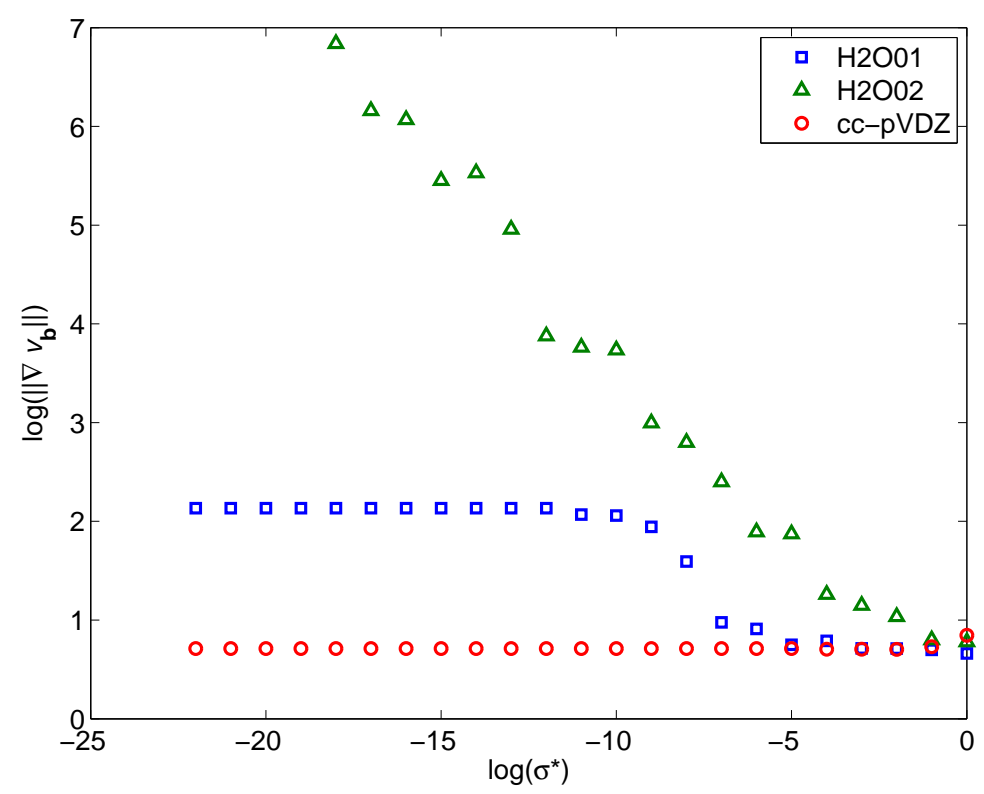

FIG. 10: TSVD regularization
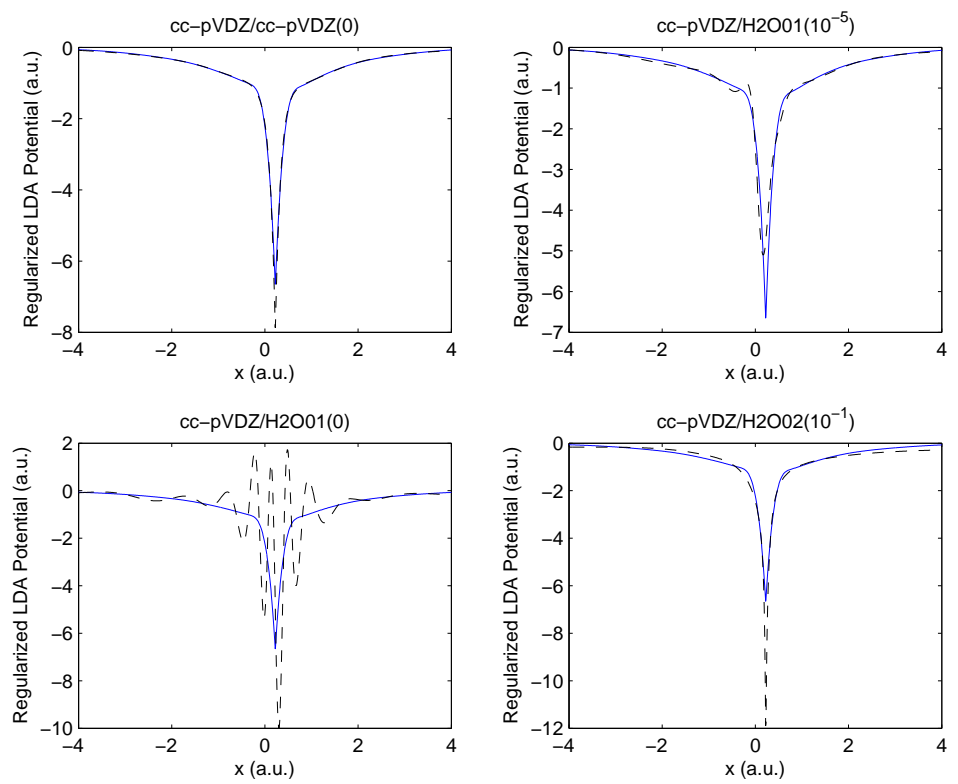

FIG. 11: TSVD-regularized LDA potentials for water (cc-pVDZ) for various values of the cutoff. The solid line depicts the exact LDA potential.

regularization. This is very encouraging given the differences in the potential basis sets. An inflexion point is well defined and gives an approximation to the OEP energy of $E^{\mathrm{OEP}}-$ $E^{\mathrm{HF}} \sim 1 \times 10^{-2.5}$ a.u.. If we want to get more accurate energies a finer $\lambda$-sampling grid would be necessary. The given basis sets can reproduce the HF energy to an agreement of $\sim 10^{-5}$ 


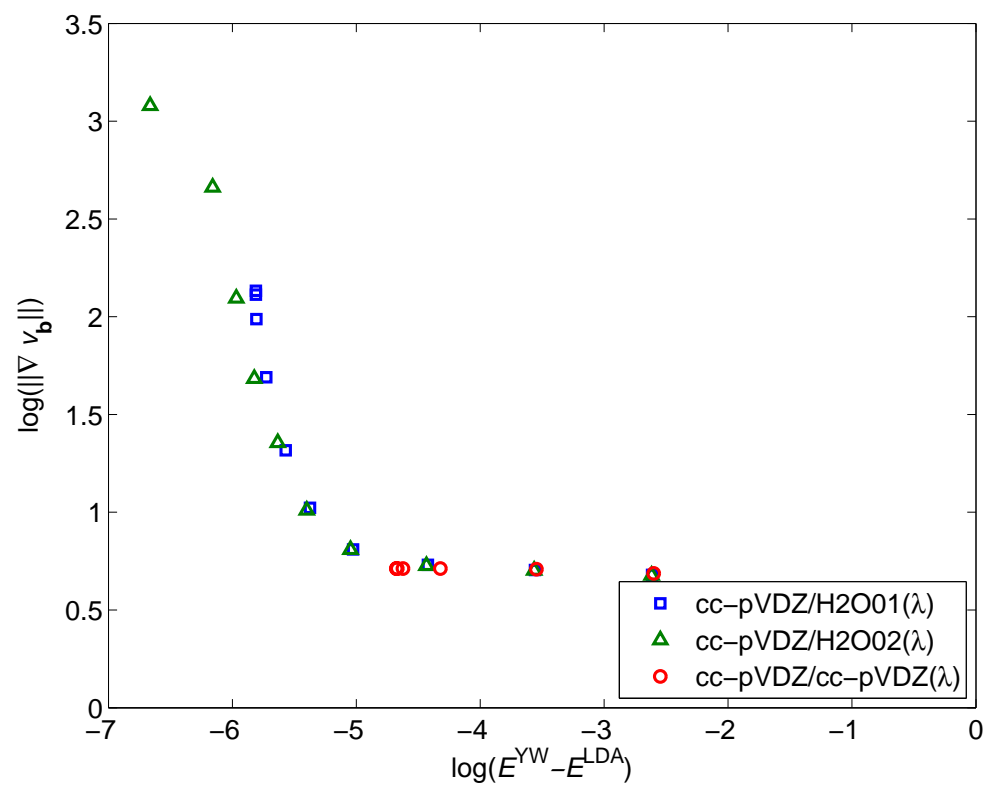

FIG. 12: LDA L-curves for water in the various potential basis sets.

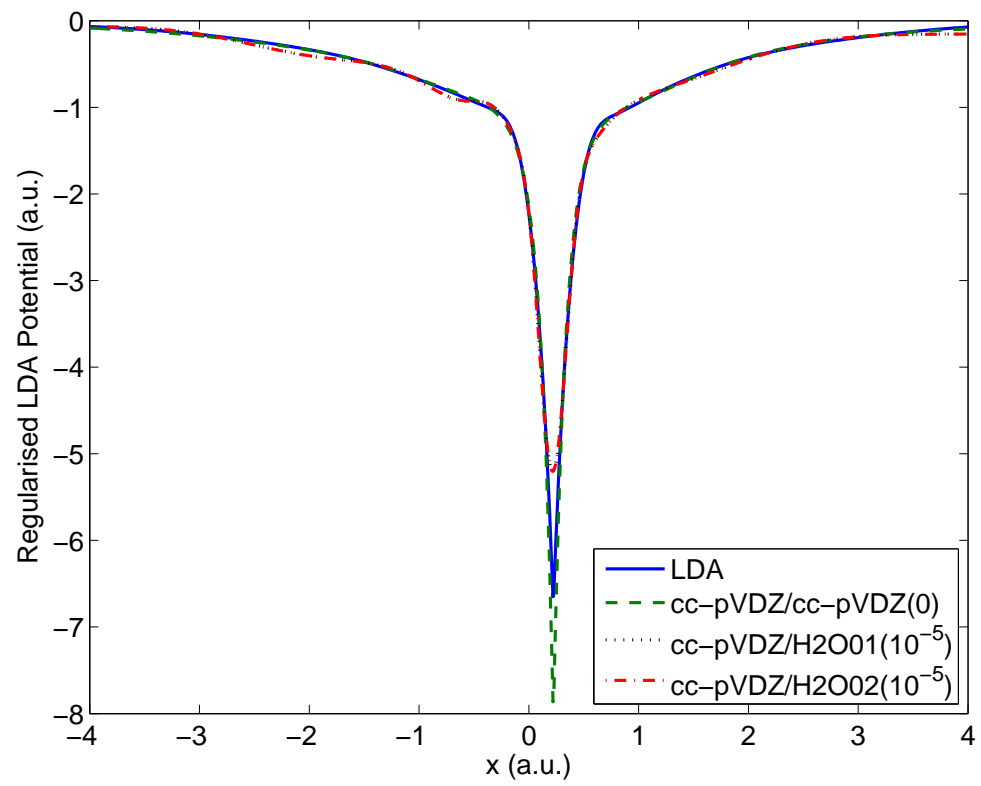

FIG. 13: Optimal LDA OEP potentials for water (cc-pVDZ) obtained from the L-curve analysis for each basis set.

a.u. with nonphysical potentials. The potentials we extract from the L-curve are shown in Fig. (18). The two ET basis sets produce essentially identical potentials, differing only at the oxygen cusp. The potential cc-pVDZ/cc-pVDZ(0) is also of an acceptable quality, but as expected with the use of basis sets designed for orbitals, it does struggle to reproduce the 


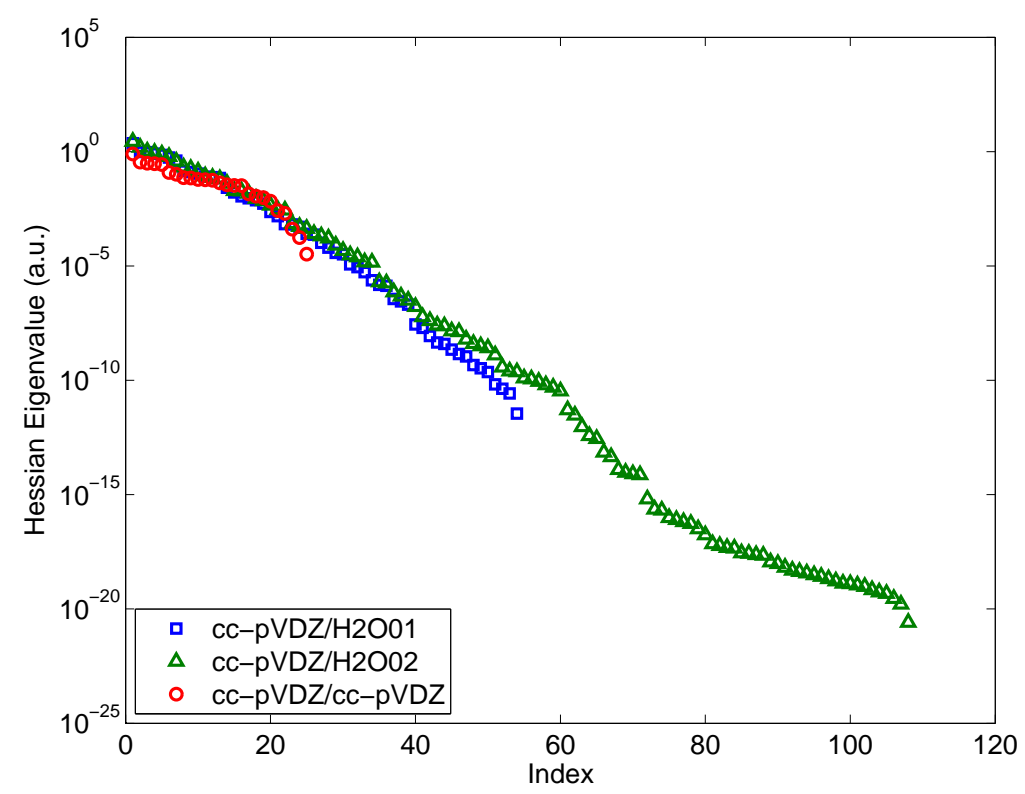

FIG. 14: Spectrum of the approximate Hessian for each basis set.

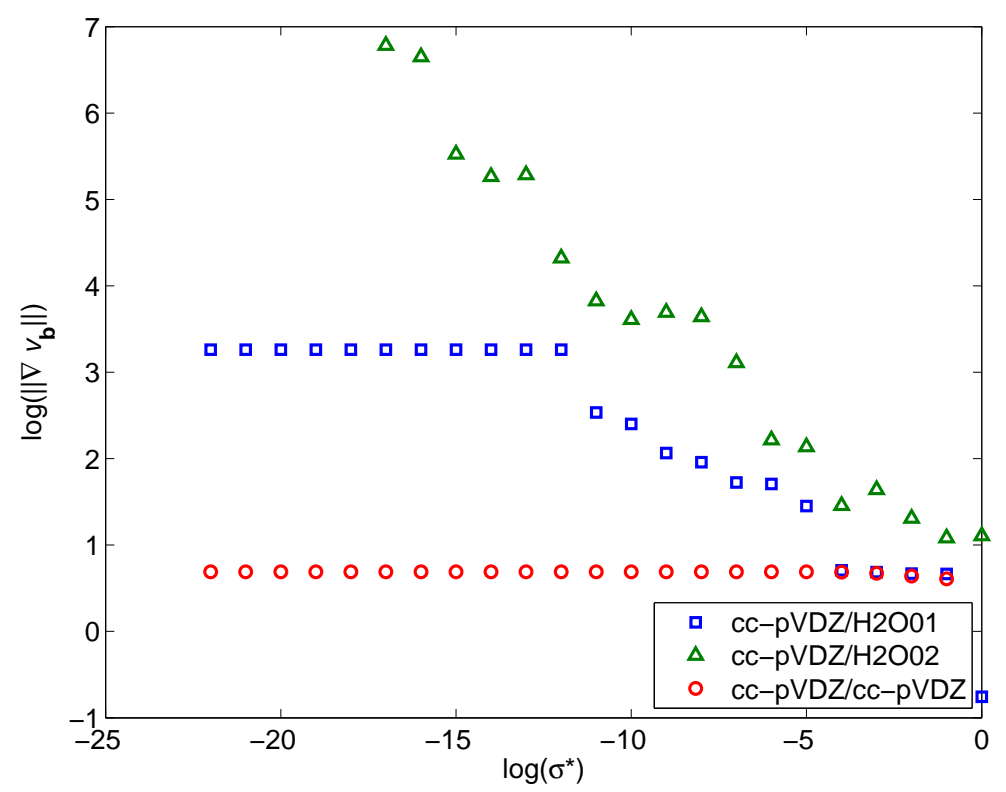

FIG. 15: TSVD regularization

finer details that a flexible ET basis can. 


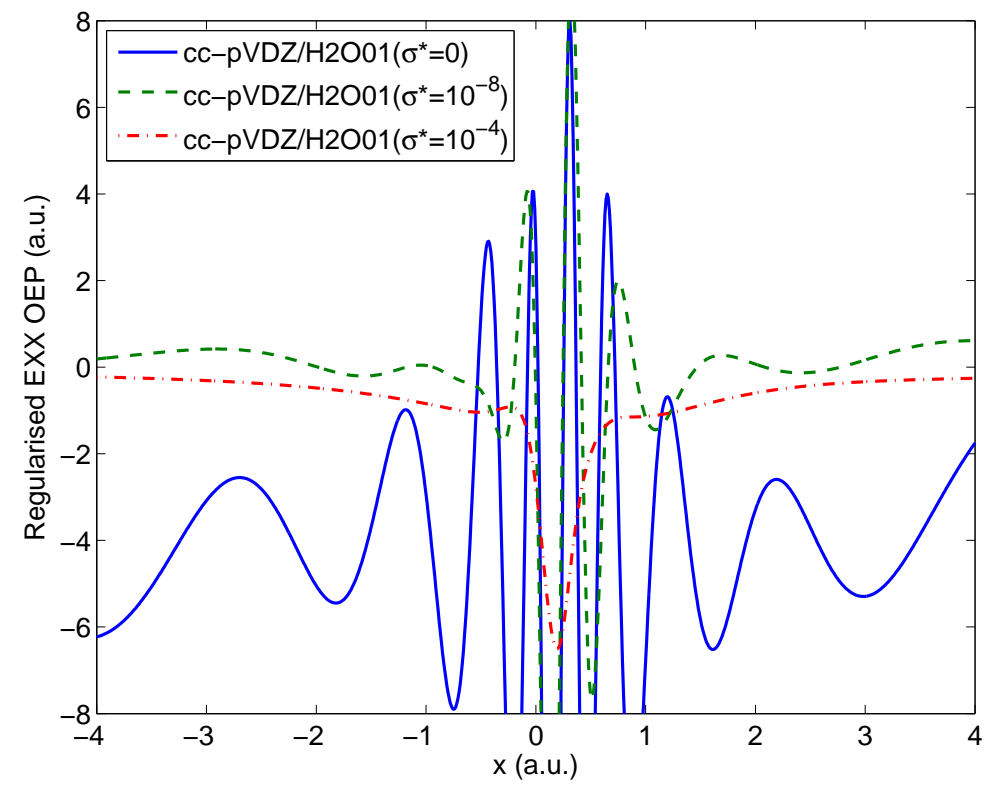

FIG. 16: TSVD-regularized EXX potentials for water (cc-pVDZ/H2O01) for various values of the TSVD cutoff

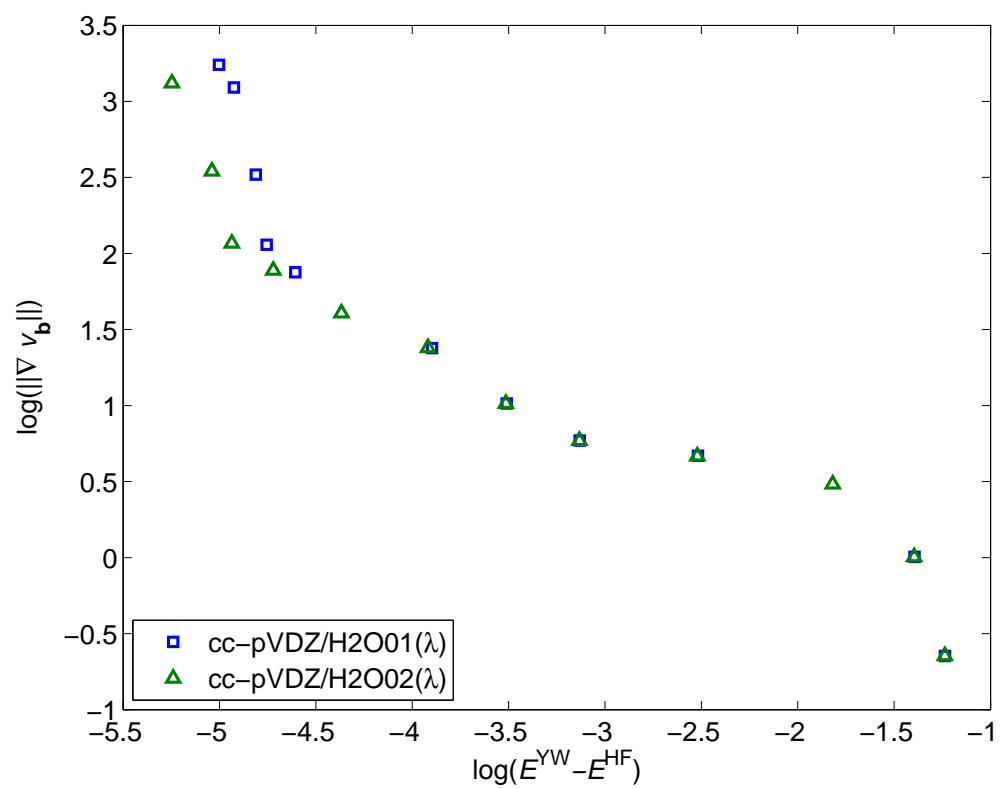

FIG. 17: LDA L-curves for water in the various potential basis sets.

\section{NITROGEN}

The potential basis sets we use for $\mathrm{N}_{2}$ are as denoted as N2256, N2512, and N28192, consisting of 13s3p, 14s5p and 18s8p uncontracted cartesian Gaussians with exponents given 


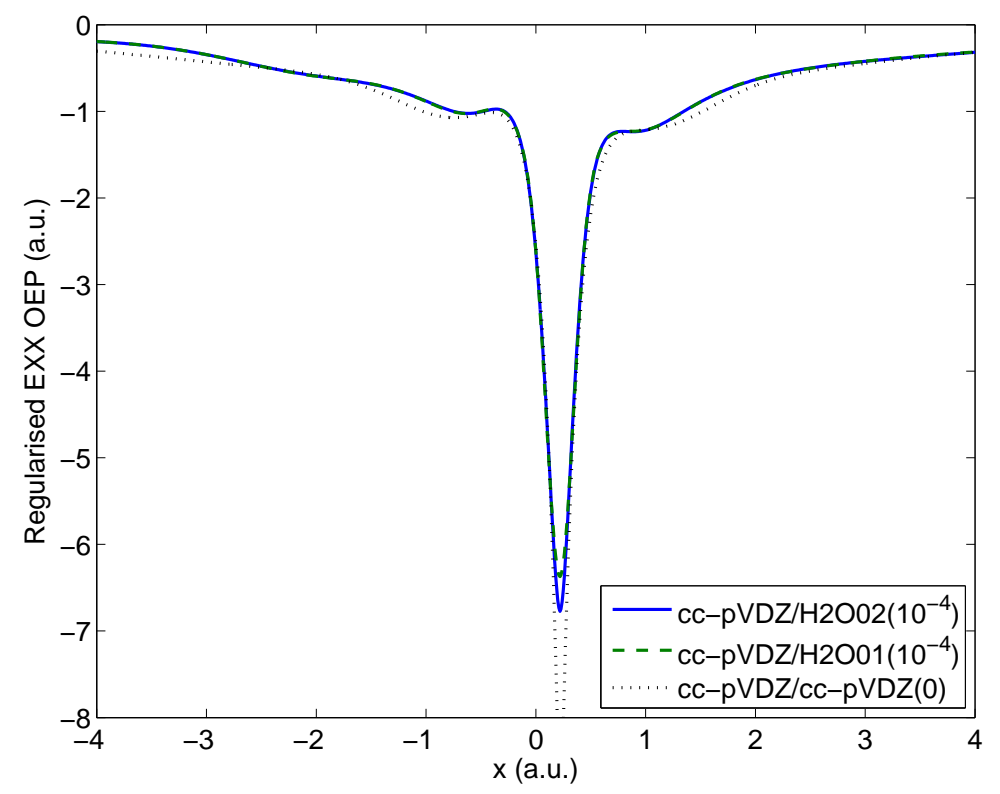

FIG. 18: Optimal EXX OEP potentials for water (cc-pVDZ) obtained from the L-curve analysis for each basis set.

in Table III. An orbital basis if cc-pVDZ is used. We use the a bond length of $1.098 \AA$, and plot potentials along the bond axis.

\begin{tabular}{c|c||l} 
& s-exponents & p-exponents \\
\hline $\mathrm{N} 2256(13 \mathrm{~s} 3 \mathrm{p})$ & $2^{n},-4 \leq n \leq 8$ & $2^{n},-2 \leq n \leq 0$ \\
$\mathrm{~N} 2512(14 \mathrm{~s} 5 \mathrm{p})$ & $2^{n},-4 \leq n \leq 9$ & $2^{n},-3 \leq n \leq 1$ \\
$\mathrm{~N} 28192(18 \mathrm{~s} 8 \mathrm{p})$ & $2^{n},-4 \leq n \leq 13$ & $2^{n},-2 \leq n \leq 5$
\end{tabular}

TABLE I: Exponents used in constructing the potential basis sets used for our $\mathrm{N}_{2}$ calculations.

\section{A. LDA}

The results for nitrogen are very much similar to those for water previously discussed. All three ET basis sets are capable of producing very oscillatory potentials. The L-curve analysis in Fig. (20) provides appropriate potentials (Fig. (21)). 


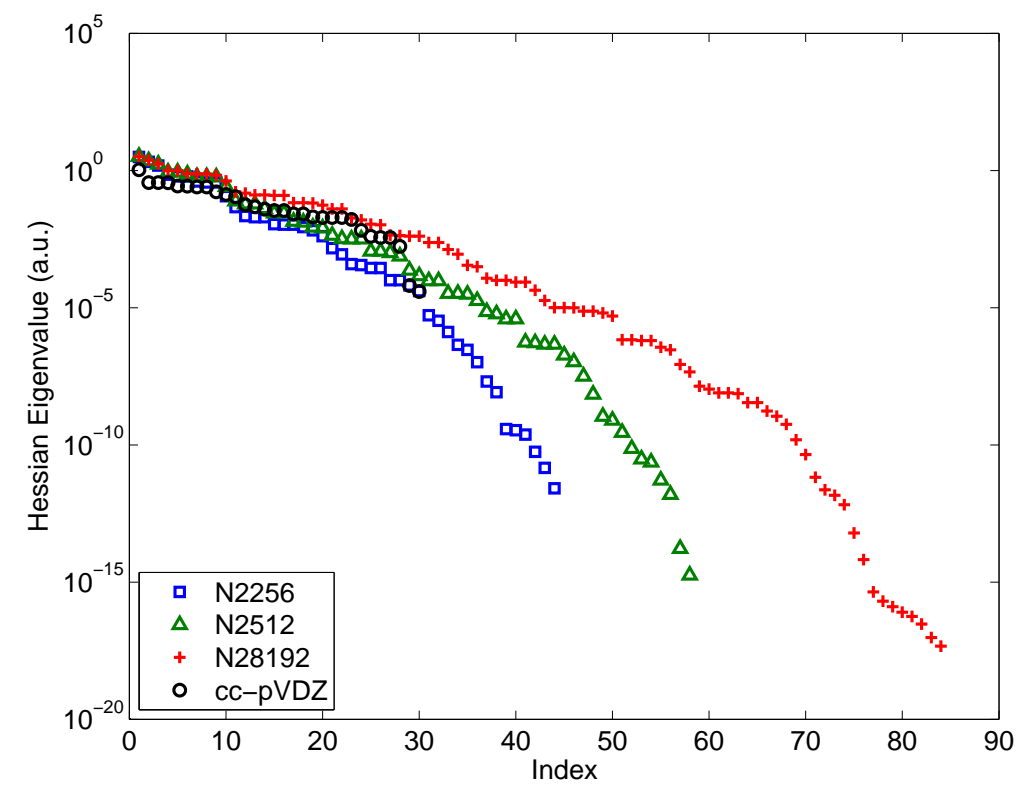

FIG. 19: Spectrum of the approximate Hessian for each basis set.

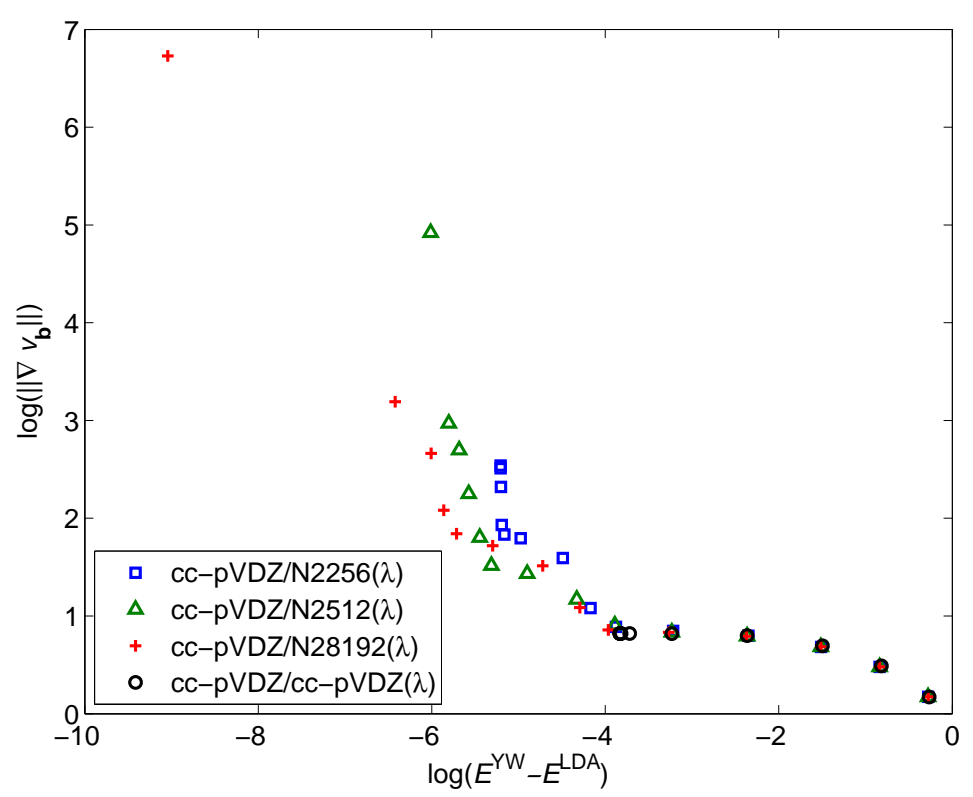

FIG. 20: $\lambda$ regularization

\section{B. EXX}

The L-curve in Fig. (23) and potentials have been presented in the text for this case. Included here is the Hessian spectrum, and examples of $\lambda$-regularized potentials for nonoptimal $\lambda$. 


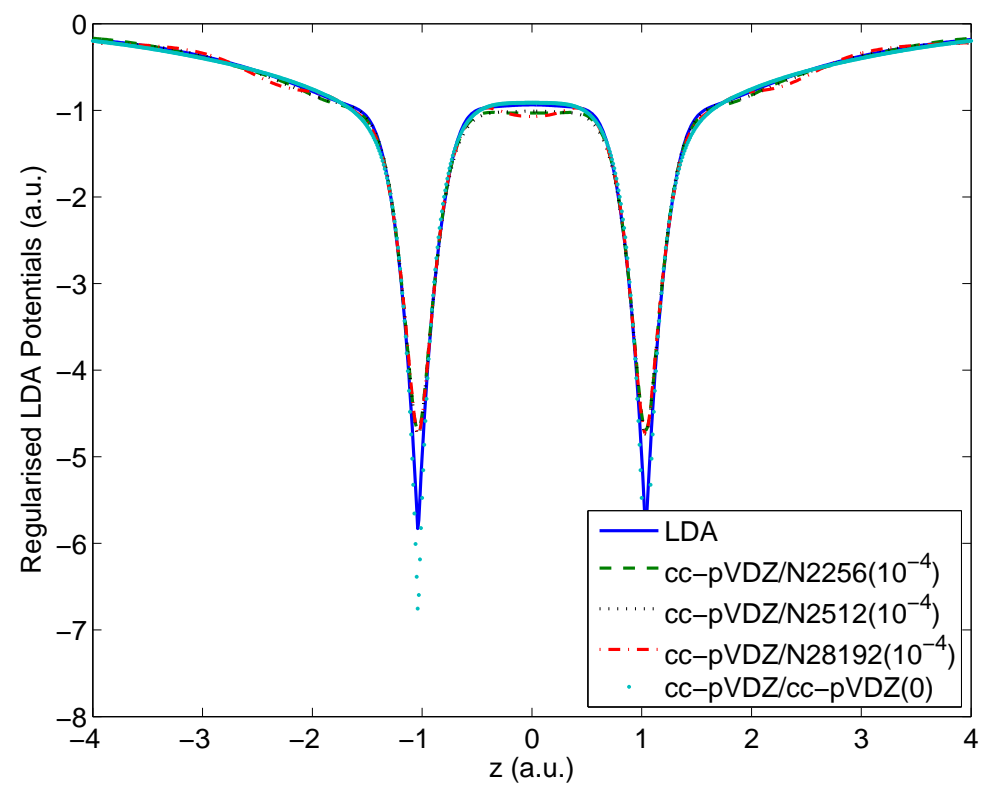

FIG. 21: Optimal LDA OEP potentials for $\mathrm{N}_{2}$ (cc-pVDZ) obtained from the L-curve analysis for each basis set.

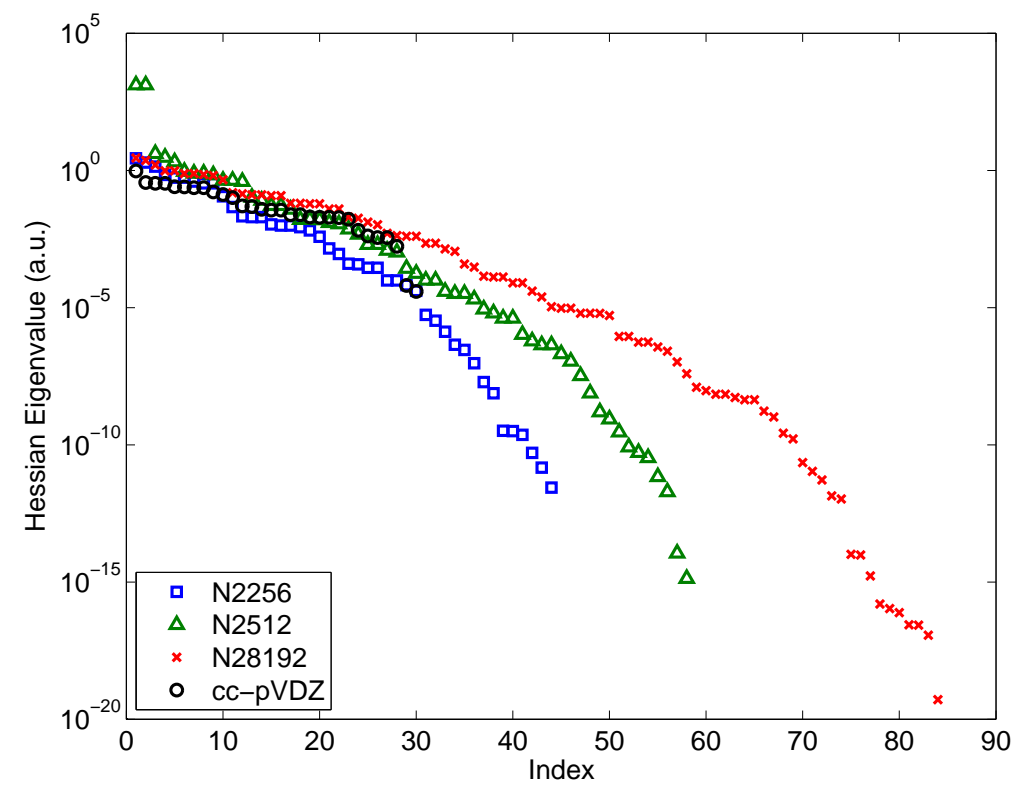

FIG. 22: Spectrum of the Approximate Hessian for each basis set. 


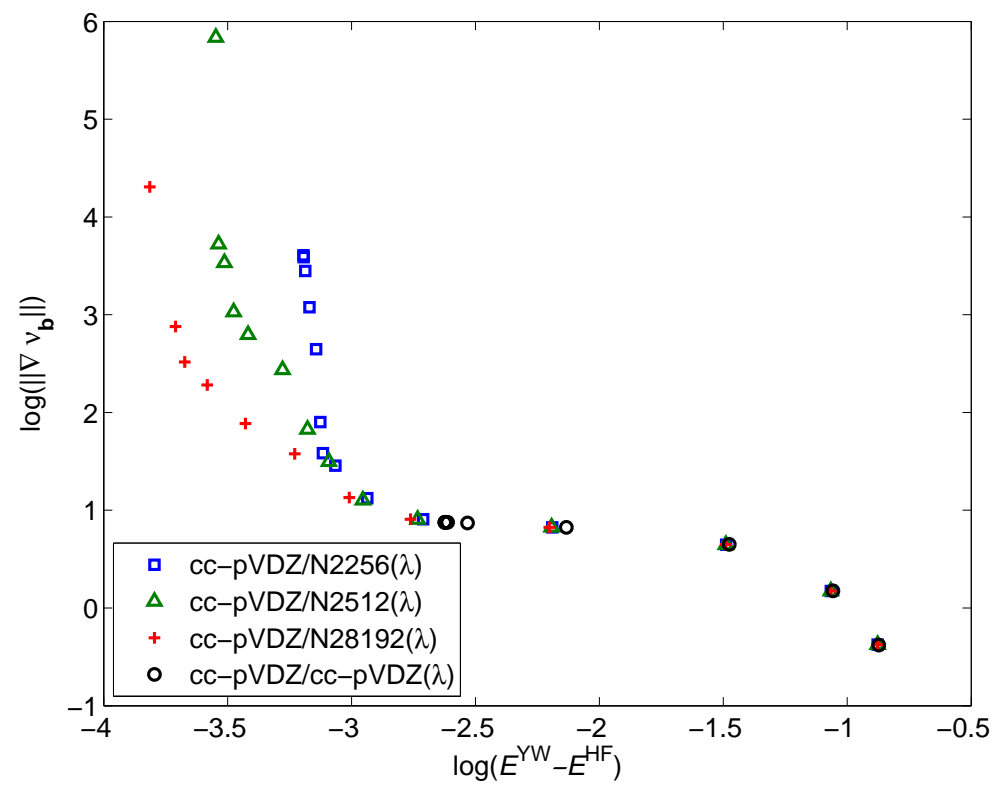

FIG. 23: EXX L-curves for $\mathrm{N}_{2}$ in the various potential basis sets.

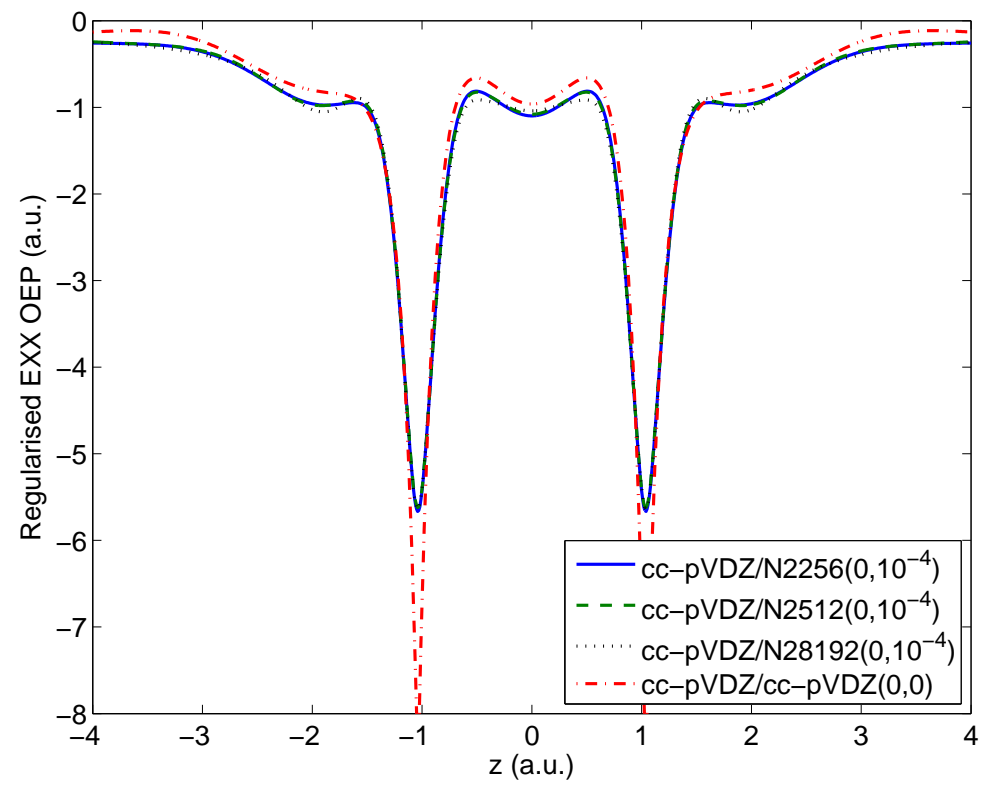

FIG. 24: Optimal EXX OEP potentials for $\mathrm{N}_{2}$ (cc-pVDZ) obtained from the L-curve analysis for each basis set. 

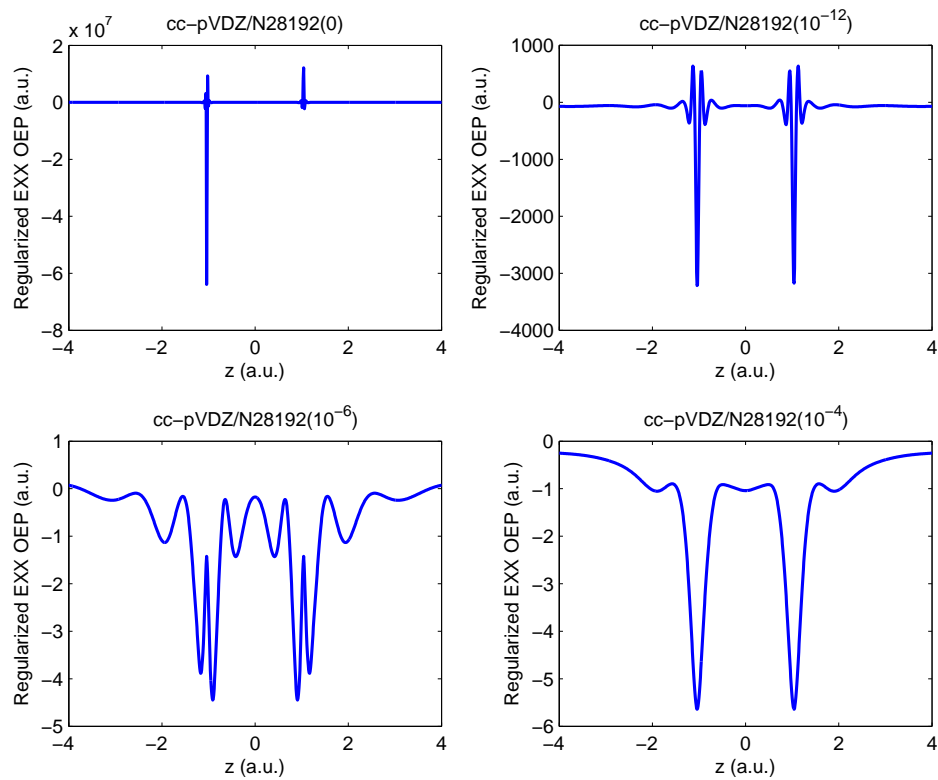

FIG. 25: $\lambda$-regularized potentials from various points on the L-curve for the calculation ccpVDZ/N2256

\section{ARGON}

\section{A. EXX}

Here we look at the effects of using a very high quality basis set, the Partridge Uncontracted 3 basis. For the potential basis set we use the Partridge Uncontracted 3 set itself, as well as a 20s ET basis Ar32768 with exponents $2^{n},-4 \leq n \leq 15$.

The L-curve of Fig. (27) is interesting as it shows an example where the use of coinciding basis sets for orbitals and potential does give rise to nonphysical potentials. This would be difficult to predict on the basis of the Hessian spectrum of Fig. 26 alone.

Also notable from the L-curve is the very well defined EXX OEP energy $\Delta E=5.2 \times 10^{-3}$ a.u.

\section{B. Small Potential Basis Sets}

Finally, we consider the situation where we use a basis set that is so small and inflexible that it is not able to provide an adequate description of the OEP. The final three figures repeat those shown in the paper for argon, however now with the inclusion of the small 


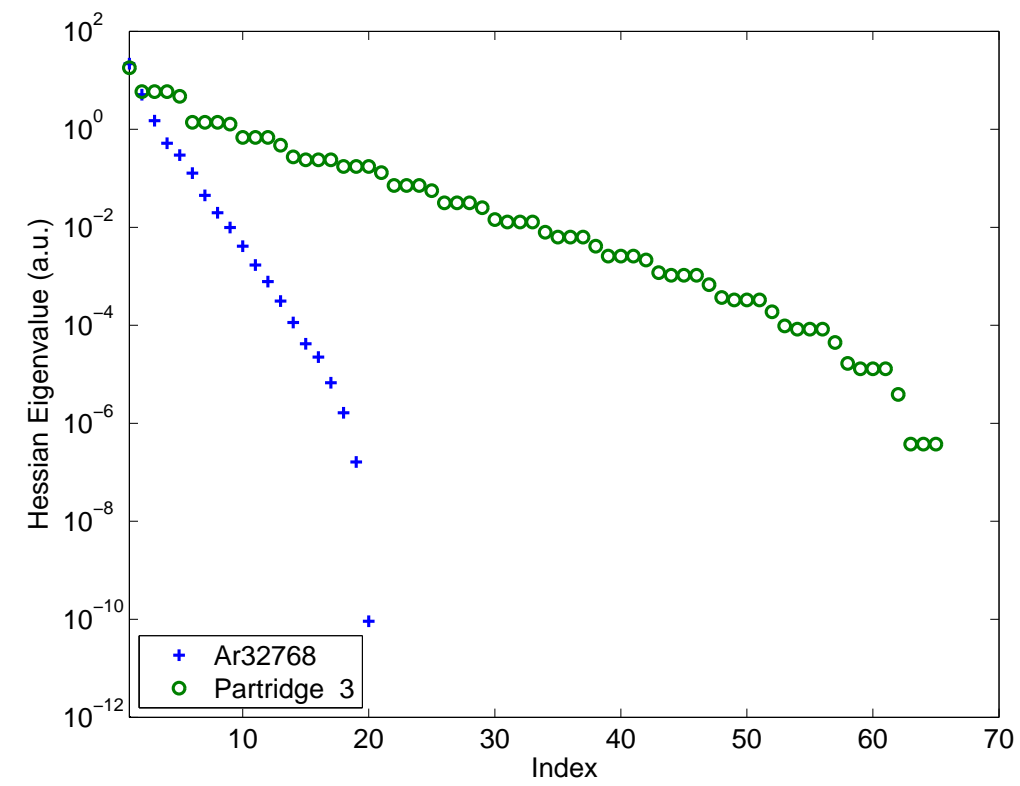

FIG. 26: Spectrum of the Approximate Hessian for each basis set.

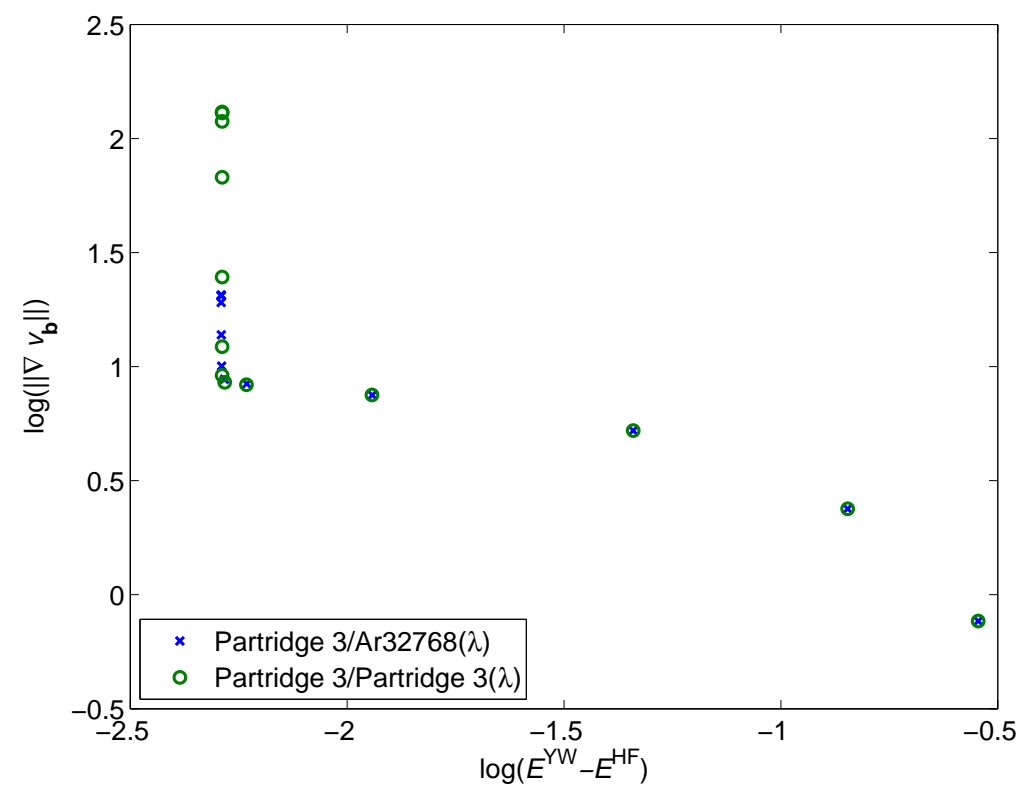

FIG. 27: EXX L-curves for Ar (Partridge Uncontracted 3)

potential basis set Ar64. While this basis set gives significantly different L-curves (Figs. (29) and (31) for LDA and EXX respectively) and potentials (Figs. (30) and (32)) from those larger basis sets, we see that the L-curve analysis still is able to generate a sensible potential differing only near the nuclei. 


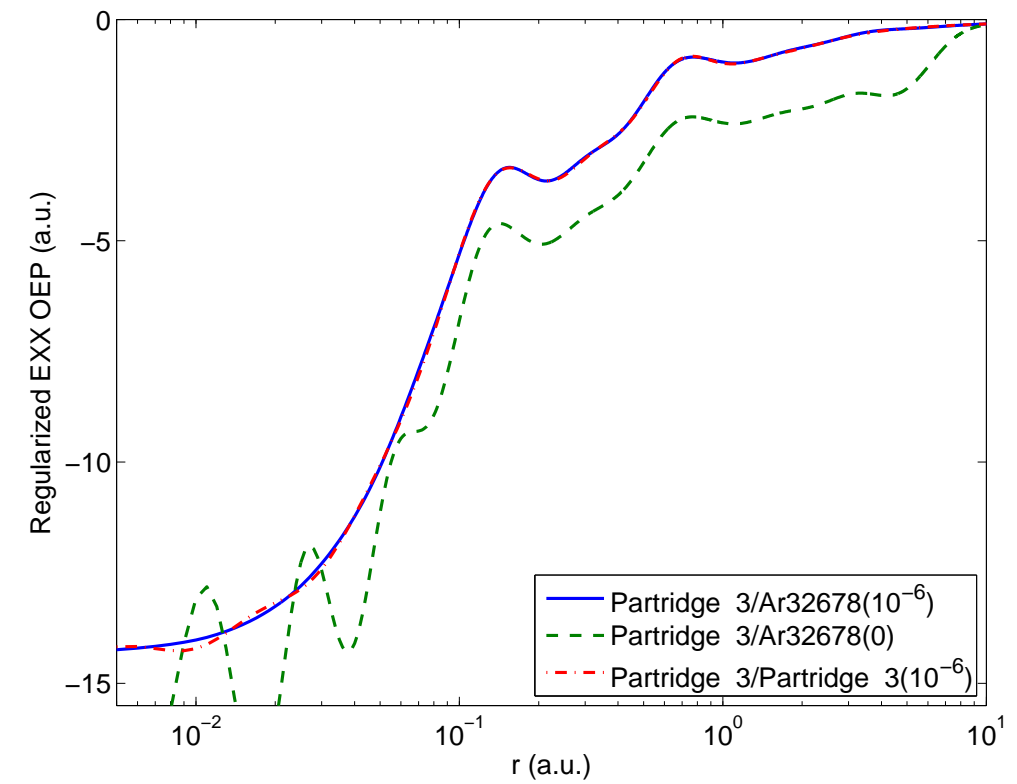

FIG. 28: Optimal regularized potentials for each basis set, and an unregularised potential

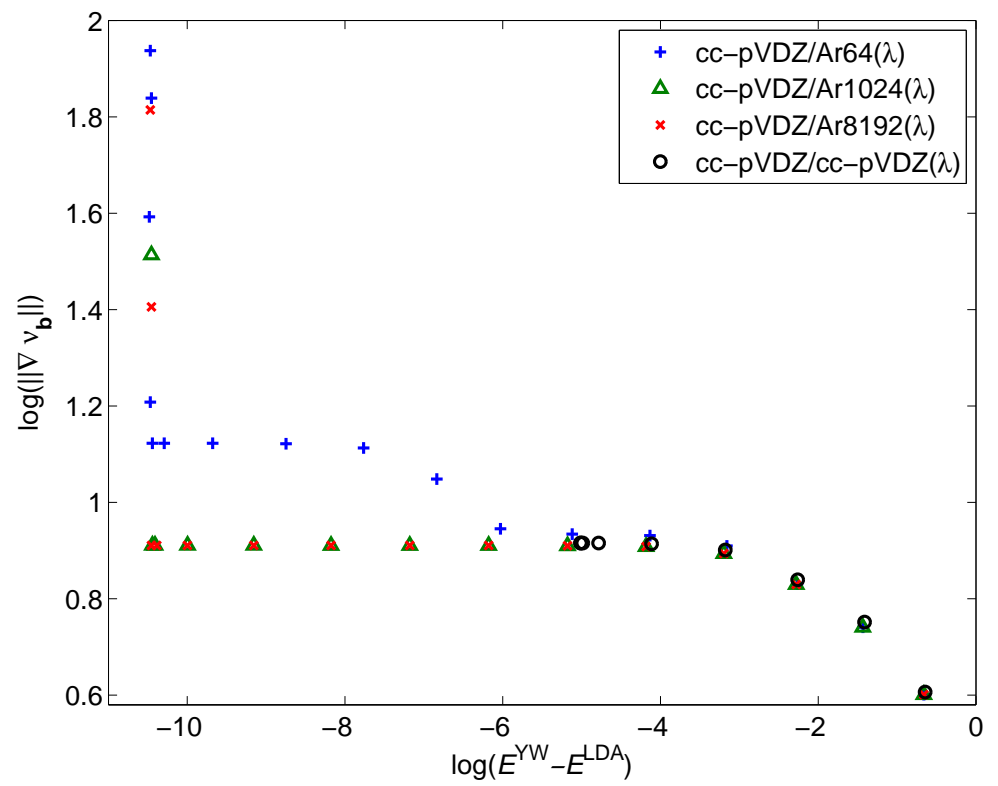

FIG. 29: LDA L-curves for Ar (cc-pVDZ) 


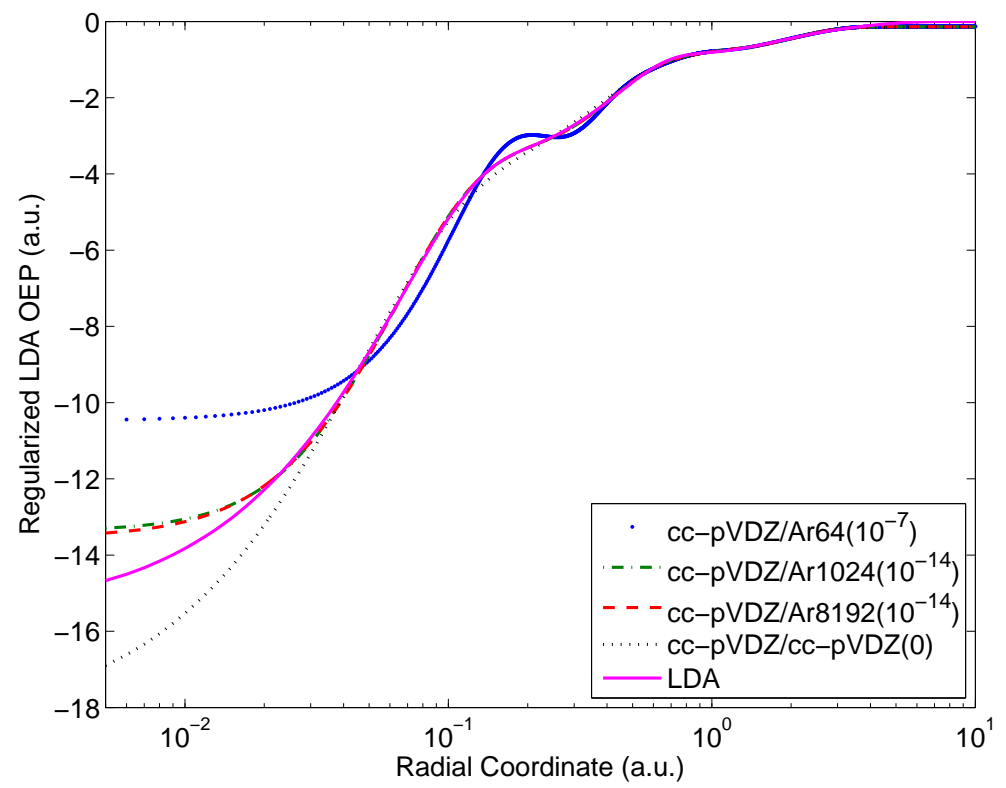

FIG. 30: Optimal regularized LDA potentials for each basis set

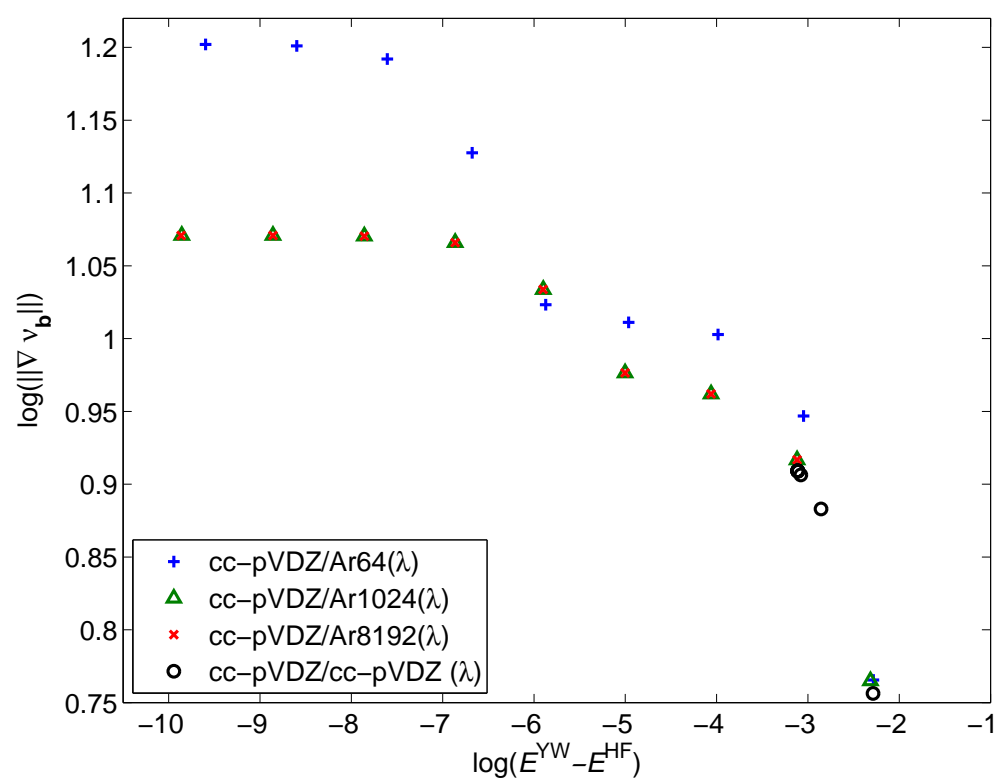

FIG. 31: EXX L-curves for Ar (cc-pVDZ) 


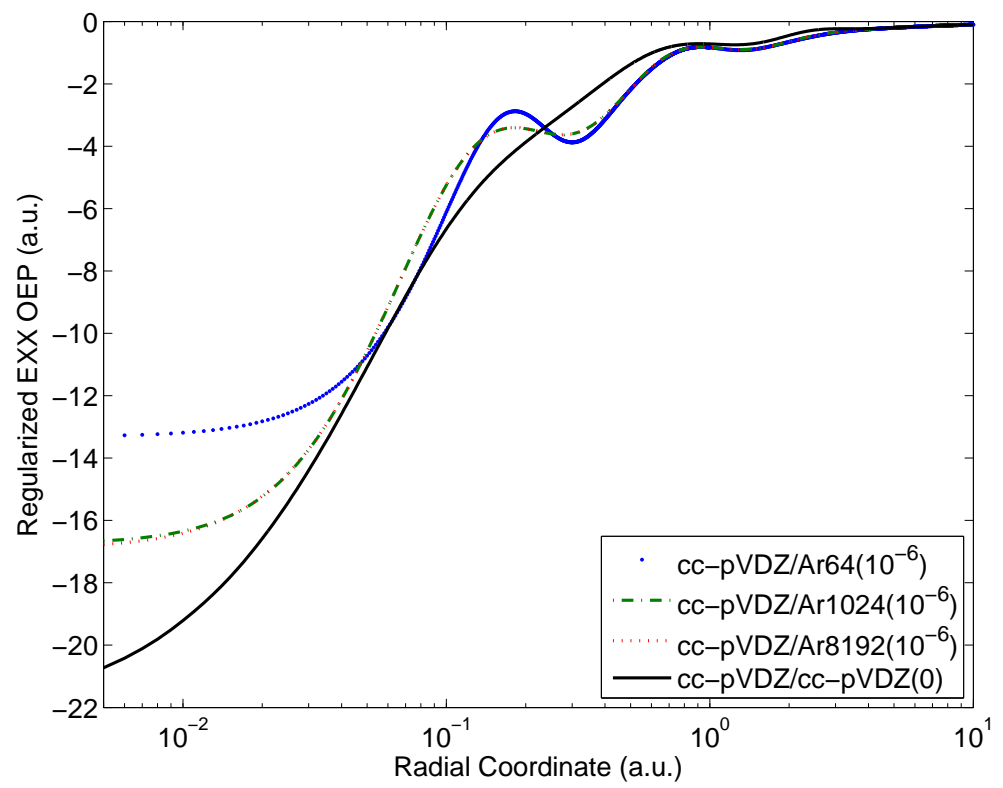

FIG. 32: Optimal regularized EXX potentials for each basis set 